\title{
Geographic location as a modifiable cardiac risk factor
}

\author{
Genevieve Gabb MBBS, Leonard Arnolda MBBS PhD
}

— Cite as: CMAJ 2017 April 3;189:E482-3. doi: 10.1503/cmaj.170116

See related article www.cmaj.ca/lookup/doi/10.1503/cmaj.160823

a $\mathrm{n}$ a linked study, Tu and colleagues found variations in cardiac health across Ontario and report an inverse association between primary care service provision and cardiac events in a population without known vascular disease. ${ }^{1}$ Geography has been central to the development of knowledge and understanding of cardiovascular disease and risk factors, its pathophysiologic basis and development of effective strategies for clinical intervention. ${ }^{2}$ Addressing regional variation is important to improve population-wide management of cardiovascular disease. But can geography be considered a modifiable variable?

The historical importance of geography in understanding cardiovascular disease is reflected in the names of early pivotal studies, such as the Minnesota Business and Professional Men's Study, ${ }^{3}$ the Seven Countries Study ${ }^{4}$ and the well-known Framingham Heart Study. ${ }^{5}$ These research efforts involved collection of study data from selected local cohorts of relatively few participants from limited geographical regions; these three studies involved less than 20000 participants, yet effectively identified key demographic (age and sex), lifestyle (smoking, diet and physical activity) and clinical (high blood pressure and cholesterol) risk factors that are largely modifiable. Interventions directed at these risk factors have contributed to global declines in cardiovascular disease.

Considerable geographic variations in both incidence of vascular disease and prevalence of important risk factors is a recognized current challenge. A 2014 review of cardiovascular epidemiology and prevention studies estimated 20 - to 30 -fold variations in rates of death for coronary heart disease between countries of low incidence, compared with countries of high incidence. ${ }^{2}$ In the United States, an excess of potentially preventable deaths in nonmetropolitan areas has been observed, not only for cardiac disease but for the five leading causes of death (i.e., heart disease, cancer, respiratory disease, stroke and unintended injury), with the gap widening over time rather than decreasing. ${ }^{6}$ Many of the leading causes of death share common risk factors. It is clear that reducing the gap in cardiac health status between regions would have widespread general health benefits.

In contrast to early cardiovascular epidemiological studies, the authors of the Cardiovascular Health in Ambulatory Care Research Team (CANHEART) Regional Variation study, which involves a large

\section{KEY POINTS}

- Effectively addressing regional variation is necessary to improve population-wide cardiovascular health.

- Approaches to address regional variation should support remote communities and delivery of primary health care; a sole focus on traditional cardiac risk factors will be ineffective.

- An innovative clinical network in South Australia has been effective in reducing regional variation in cardiac disease, across a large, sparsely populated area.

population-based cohort of about 5.5 million community-dwelling adults in Ontario, use routinely collected population data from existing databases (i.e., sociodemographics, cardiovascular risk factors, health care service and medication use, and clinical outcomes). ${ }^{1}$ The authors provide data in an appendix that show an almost twofold variation in rates of cardiovascular events between regions of lowest and highest incidence: from 2.1 events per 1000 person-years for women in low-risk regions to 7.7 per 1000 person-years for men in high-risk regions. Variation was consistent across men and women. Although most of the variation (about 75\%) could be attributed to demographic and socioeconomic factors, ethnic composition and traditional cardiac risk factors, health system factors alone account for about $16 \%$ of this variation in cardiovascular disease events between local health networks. Patients in the areas with the highest event rates mainly remote and sparsely populated areas - had fewer visits to family physicians, less lipid screening, poorer blood pressure control and less use of statins in older adults with diabetes.

Other jurisdictions have faced similar challenges. Geographic variation in cardiac disease is found across Australia, with higher rates in rural, regional and remote areas. ${ }^{7}$ South Australia, a mainland state, is similar in size to Ontario but has a smaller population (1.7 million). About $75 \%$ of South Australia's population lives in the state capital, with the remaining inhabitants scattered across an area of $1000000 \mathrm{~km}^{2}$, which presents a challenge to the provision of a modern, effective health service. To address worse outcomes for cardiac events in remote regions, the Inte- 
grated Cardiovascular Clinical Network (iCCnet Country Health South Australia) ${ }^{8}$ was created in South Australia in 2001, initially to help primary care practitioners manage acute coronary syndromes occurring in this rural and remote population. ${ }^{9}$ In 2013 , the network started the Country access to cardiac health (CATCH) program to improve secondary prevention after postacute care. ${ }^{10}$ Both programs have been effective: the difference in cardiac mortality after an acute event between rural and metropolitan areas was eliminated by 2010 , and there was a substantial reduction in readmissions of patients from rural areas.

Although iCCnet originated from efforts to improve outcomes for acute coronary events rather than being directed at primary prevention, which is arguably more complex than managing acute events, iCCnet may provide insight that is broadly applicable to cardiovascular health in areas where remoteness and distance are important. Strong clinical leadership, commitment to implementation of evidence and continuous improvement, and use of innovative models (e.g., telehealth and point-of-care testing) to support existing primary care practitioners have been particularly important, as well as acknowledging the issues of working across vast, sparsely populated areas with limited resources. Building relationships between services and providers has improved response times to urgent events. Although managing risk factor decisions is not as urgent as acute coronary syndromes, building strong relationships based on a shared understanding among care providers that addressing cardiac risk effectively and expeditiously is important.

However, the solution to reducing variations in geographic incidence of primary cardiac events will not be found solely in addressing health service factors. Consideration of public health measures and addressing inequalities in social determinants of health are also essential. Disease burden should be considered when determining resource allocation. We can look to lowresource settings, where unmet need in primary health care has been addressed innovatively by training and deploying nonprofessional lay counselors, ${ }^{11,12}$ for approaches that could be adapted to local contexts.

Given the problems of distance and remoteness across Ontario, effective interventions are likely to require innovative approaches that support both primary care practitioners and remote communities.

The challenge arising from the CANHEART study is how to move from observation of difference to effective interventions. An approach that focuses solely on traditional clinical risk factors is unlikely to be effective. Addressing geographic variation is difficult, but inaction is not acceptable. The experience in South Australia is reason for optimism. Innovative clinical networks and creative efforts to address unmet need could be part of the solu- tion. As with addressing traditional clinical cardiac risk factors, repeated measures that include considerations of geography will be important to monitor progress and will have potential as a future use of routinely collected population data.

\section{References}

1. Tu JV, Chu A, Maclagan L, et al. Regional variations in ambulatory care and incidence of cardiovascular events. CMAJ 2017;189:E494-501.

2. Wong ND. Epidemiological studies of $\mathrm{CHD}$ and the evolution of preventive cardiology. Nat Rev Cardiol 2014;11:276-89.

3. Keys A, Taylor HL, Blackburn H, et al. Coronary heart disease among Minnesota business and professional men followed fifteen years. Circulation 1963;28:381-95.

4. Keys A. Seven Countries: a multivariate analysis of death and coronary heart disease. Cambridge (MA): Harvard University Press; 1980.

5. Dawber TR, Meadors GF, Moore FE. Epidemiological approaches to heart disease: the Framingham Study. Am J Public Health Nations Health 1951;41:279-81.

6. Moy E, Garcia MC, Bastian B, et al. Leading causes of death in nonmetropolitan and metropolitan areas - United States, 1999-2014. MMWR Surveill Summ 2017;66:1-8.

7. Rural, regional and remote health: a study on mortality. 2nd ed. Canberra: Australian Institute of Health and Welfare; 2007.

8. Integrated Cardiovascular Clinical Network CHSA [home page]. Bedford Park: iCCnet CHSA. Available: www.iccnetsa.org.au (accessed 2017 Jan. 26).

9. Tideman PA, Tirimacco R, Senior DP, et al. Impact of a regionalized clinical cardiac support network on mortality among rural patients with myocardial infarction. Med J Aust 2014;200:157-60.

10. Tideman PA, Tirimacco R, Berry $\mathrm{N}$ et al. Country access to cardiac health (CATCH) program: improving rehospitalisation rates and length of stay for cardiac rehabilitation using innovative telehealth delivery. Heart Lung Circ 2015; 24(Suppl 3):S453.

11. Patel V, Weobong B, Weiss H, et al. The Healthy Activity Program (HAP), a lay ounsellor-delivered brief psychological treatment for severe depression, in primary care in India: a randomized controlled trial. Lancet 2017;389:176-85.

12. Nadkarni A, Weobong B, Weiss $\mathrm{H}$, et al. Counselling for Alcohol Problems (CAP), a lay counsellor-delivered brief psychological treatment for harmful drinking in men, in primary care in India: a randomized controlled trial. Lancet 2017; 389:186-95.

Competing interests: Genevieve Gabb has received speaker fees from AstraZeneca and Pfizer, and consultant fees from Therapeutic Goods Administration, Canberra, Australia, and Adelaide Health Technology Assessment. Leonard Arnolda has received a speaker fee from Pfizer and nonfinancial support for travel from the National Heart Foundation of Australia.

Affiliations: Royal Adelaide Hospital (Gabb), University of Adelaide, Adelaide, Australia; Illawarra Health and Medical Research Institute (Arnolda), University of Wollongong, New South Wales, Australia

Contributors: Both authors contributed equally and substantially to the article, drafted and revised the work critically for important intellectual content, gave final approval of the version to be published and agreed to be accountable for all aspects of the work.

This article was solicited and has not been peer reviewed.

Correspondence to: Genevieve Gabb, genevieve.gabb@sa.gov.au 